\title{
Ginseng gintonin attenuates the disruptions of brain microvascular permeability and microvascular endothelium junctional proteins in an APPswe/PSEN-1 double-transgenic mouse model of Alzheimer's disease
}

\author{
MINHEE JANG ${ }^{1 *}$, SUN-HYE CHOI ${ }^{2 *}$, JONG HEE CHOI ${ }^{1}$, JINHEE OH ${ }^{1}$, RA MI LEE ${ }^{2}$, NA-EUN LEE ${ }^{2}$, \\ YEON-JIN CHO ${ }^{2}$, HYEWHON RHIM $^{3}$, HYOUNG-CHUN KIM ${ }^{4}$, IK-HYUN CHO ${ }^{1,5}$ and SEUNG-YEOL NAH ${ }^{2}$ \\ ${ }^{1}$ Department of Convergence Medical Science, College of Korean Medicine, Kyung Hee University, Seoul 02447; \\ ${ }^{2}$ Ginsentology Research Laboratory and Department of Physiology, College of Veterinary Medicine, \\ Konkuk University, Seoul 05029; ${ }^{3}$ Center for Neuroscience, Korea Institute of Science and Technology, Seoul 02792; \\ ${ }^{4}$ Neuropsychopharmacology and Toxicology Program, College of Pharmacy, Kangwon National University, \\ Chuncheon, Gangwon-do 24341; ${ }^{5}$ Brain Korea 21 Plus Program and Institute of Korean Medicine, \\ College of Korean Medicine, Kyung Hee University, Seoul 02447, Republic of Korea
}

Received December 10, 2019; Accepted July 30, 2020

DOI: $10.3892 / \mathrm{etm} .2021 .9741$

\begin{abstract}
It has been previously indicated that gintonin, which is a novel exogenous ginseng-derived lysophosphatidic acid (LPA) receptor ligand, restores memory dysfunctions in an APPswe/PSEN-1 double-transgenic mouse model of Alzheimer's disease (AD Tg mice) by attenuating $\beta$-amyloid plaque deposition, recovering cholinergic dysfunctions and upregulating hippocampal neurogenesis in the cortex and hippocampus. Although $\beta$-amyloid plaque depositions in $\mathrm{AD}$ is accompanied with disruptions of brain microvessels, including the brain-blood barrier (BBB), it is unknown whether gintonin exerts protective effects on brain microvascular dysfunctions in AD Tg mice. In the present study, the
\end{abstract}

Correspondence to: Professor Ik-Hyun Cho, Department of Convergence Medical Science, College of Korean Medicine, Kyung Hee University, 26 Kyungheedae-ro, Dongdaemun-gu, Seoul 02447, Republic of Korea

E-mail: ihcho@khu.ac.kr

Professor Seung-Yeol Nah, Ginsentology Research Laboratory and Department of Physiology, College of Veterinary Medicine, Konkuk University, 120 Neungdong-ro, Seoul 05029, Republic of Korea E-mail: synah@konkuk.ac.kr

*Contributed equally

Abbreviations: AD, Alzheimer's disease; BBB, brain-blood barrier; LPA, Lysophosphatidic acid; PECAM-1, platelet endothelial cell adhesion molecule-1; VEGF, vascular endothelial growth factors; ZO-1, zonula occludens-1

Key words: Ginseng, gintonin, Alzheimer's disease, brain microvessels, blood-brain barrier effects of gintonin-enriched fraction (GEF) on the changes in $\beta$-amyloid plaque depositions, brain permeability of Evans blue, and microvascular junctional proteins were investigated in $\mathrm{AD}$ Tg mice. Long-term oral administration of GEF reduced $\beta$-amyloid plaque depositions in the cortex and hippocampus of $\mathrm{AD} \mathrm{Tg}$ mice. GEF treatment also reduced the permeability of Evans blue through BBB and decreased immunoreactivity of platelet endothelial cell adhesion molecule-1 (a marker of BBB disruption) in the cortex and hippocampus of AD Tg mice in a dose-dependent manner. However, GEF elevated the protein expression of occludin, claudin-5 and zonula occludens- 1 , which are tight-junction proteins. The present results demonstrated that long-term oral GEF treatment not only attenuates $\beta$-amyloid plaque depositions in the brain but also exhibits protective effects against microvascular disruptions in $\mathrm{AD} \mathrm{Tg}$ mice. Finally, GEF exhibits anti-AD effects through attenuation of $\beta$-amyloid plaque depositions and protection against brain microvascular damage in an AD animal model.

\section{Introduction}

Lysophosphatidic acid (LPA, 1-acyl-2-hydroxy-sn-glycero3-phosphate) is a simple phospholipid present in the animal system, having diverse in vitro and in vivo biological effects in the neuronal and non-neuronal cells and organs as a lipid-derived growth factor (1). The primary cellular actions of LPA include intracellular calcium concentration or $\left[\mathrm{Ca}^{2+}\right]$ i transient induction as well as $\mathrm{Ca}^{2+}$-mediated cell proliferation, differentiation, morphological changes, migration, and survival (1). These effects are mediated by the activation of G protein-coupled LPA receptors (LPARs), of which there are six different subtypes (2). Early in vitro and in vivo studies have shown that LPA1 receptor is involved in the early brain development and cortical growth through neurogenesis (3). Recent studies have demonstrated that LPA-LPA1 
receptor axis are also involved in many adult brain functions, including emotion and cognition (4). Further, LPAR1-6 is also expressed in the endothelial cells of blood vessel $(5,6)$. LPA exists in different body fluids, such as plasma, and it takes part in angiogenesis $(7,8)$. Although LPA plays an important role in angiogenesis, little is known on the effects of LPA on maintenance of microvascular integrity including blood-brain barrier (BBB) in Alzheimer's disease (AD). Although accumulating evidence shows that $\mathrm{AD}$ patients exhibit $\mathrm{BBB}$ leakage due to alterations of BBB permeability (9-11), the main causes of vascular dysfunctions in AD are due to $\beta$-amyloid accumulation in the brain (12). It is currently unknown whether LPA affects endothelial cell junctional proteins that regulate $\mathrm{BBB}$ functions.

Panax ginseng C.A. Meyer is one of medicinal plant having diverse biological effects. Recent reports show that ginseng contains a novel exogenous LPAR ligand, gintonin (13), since gintonin includes a large amount of LPAs such as LPA $\mathrm{C}_{18: 2}$, LPA $\mathrm{C}_{18: 1}$, and LPA $\mathrm{C}_{16: 0}$, as an biologically active ingredients (14). At the cellular level, gintonin induces intracellular $\mathrm{Ca}^{2+}$ transients with low effective concentration 50 values in cells expressing LPAR1, LPAR2, LPAR3 or LPAR5, but with high effective concentration 50 in cells expressing LPAR4 and LPAR6, indicating that gintonin exhibits a differential affinity for LPARs (13). Previously, we have demonstrated that gintonin regulates various $\mathrm{Ca}^{2+}$-dependent ion channels and receptors (14). Furthermore, short-term treatment with gintonin has been found to enhance synaptic transmission by stimulating glutamate release in hippocampal slices and increase long-term potentiation (15). An in vivo study on normal mice demonstrated that long-term administration of gintonin enhanced hippocampus-dependent memory (16). Previous reports also showed that long-term treatment of gintonin attenuated $\beta$-amyloid plaque deposition in the cortex and hippocampus and restored $\beta$-amyloid-induced memory dysfunction. Moreover, long-term administration of gintonin-enriched fraction (GEF) also restored $\beta$-amyloid-induced cholinergic brain dysfunction by increasing acetylcholine synthesis and stimulating hippocampal neurogenesis in an APPswe/PSEN-1 double-Tg mouse model of AD (AD Tg mice) (16-18). Although LPA is involved in brain functions in normal and AD animal model, it is unknown whether gintonin can protect the brain microvessels from damages induced by $\beta$-amyloid plaque accumulation in a Tg mouse model of AD.

In the present study, we investigated the effects of GEF on $\beta$-amyloid plaque depositions, disruptions of brain microvascular permeability, and microvascular endothelial cell protein expression in the brain of AD Tg mice. We found that long-term oral administration of GEF attenuated depositions of $\beta$-amyloid plaques, the increased Evans blue permeability, reduced expression of platelet endothelial cell adhesion molecule (PECAM-1), and enhanced occludin, claudin-5, and zonula occludens ( $\mathrm{ZO})-1$ in the cortex and hippocampus of AD Tg mice. We further discussed the pharmacological roles of GEF on brain microvascular integrity in AD.

\section{Materials and methods}

GEF preparation. Four-year-old Korean ginsengs were purchased from local ginseng market (Geumsan Ginseng
Cooperative, Geumsan, Republic of Korea) and identified and the specimen (voucher no. NIBRVP0000730014) was deposited at the herbarium of the National Institute of Biological Resources (Herbarium code: KB, http://sweetgum. nybg.org/science/ih/herbarium-details/?irn=138656). GEF was prepared as described previously (19). One kilogram of ginseng was ground into small pieces $(>3 \mathrm{~mm})$ and refluxed eight times with $70 \%$ fermented ethanol for $8 \mathrm{~h}$ at $80^{\circ} \mathrm{C}$. The extract (350 g) was concentrated, dissolved in distilled cold water at a ratio of 1 to 10 , and stored at $4^{\circ} \mathrm{C}$ for $24-96 \mathrm{~h}$. The supernatant and precipitate from the water fractionation after ethanol extraction was separated by centrifugation at $3,000 \mathrm{rpm}$ for $20 \mathrm{~min}$. After centrifugation, the precipitate was lyophilized.

The main active ingredient compositions of GEF. The detailed compositions of this fraction including LPAs as an active ingredient of GEF were identified in previous report through LC-MS/MS analysis (20). Thus, GEF contains $\sim 7.5 \%$ linoleic $\left(\mathrm{C}_{18: 2}\right), 2.8 \%$ palmitic $\left(\mathrm{C}_{16: 0}\right)$, and $1.5 \%$ oleic acids $\left(\mathrm{C}_{18: 1}\right)$. GEF contains $\sim 0.2 \%$ LPA $\mathrm{C}_{18: 2}, 0.06 \%$ LPA $\mathrm{C}_{16: 0}$, and $0.02 \%$ LPA $\mathrm{C}_{18: 1}$. GEF contains $0.08 \%$ lysophosphatidylcholine, $0.03 \%$ lysophosphatidylethanolamine, and $0.13 \%$ lysophosphatidylinositols. GEF also contains $\sim 1 \%$ phosphatidic acid (PA) 16:0-18:2, 0.5\% PA 18:2-18:2, and 0.2\% PA 16:0-18:1 (20).

Animals, drug treatments, and ethical approval. The breeding pairs of double-Tg male mice expressing the mutant swe-A $\beta P P$ (AßPPswe) and mutant presenilin-1 (PSEN-1) genes (deletion of exon 9) [A $\beta$ PPswe/PSEN-1 double-Tg mice, B6C3-Tg (A $\beta$ PPswe/PSEN1dE9) 85Dbo/J; The Jackson Laboratory] were housed and bred in an approved animal facility at the Kangwon National University, Republic of Korea. Four animals were housed in each cage under constant temperature $\left(23 \pm 1^{\circ} \mathrm{C}\right)$ and humidity $(55 \pm 5 \%)$. The animals were provided access to food and water ad libitum and were maintained under a 12-h light/dark cycle (lights on 07:00-19:00). The mice were divided into wild-type (WT, $n=8), \operatorname{AD} \operatorname{Tg}(n=8)$, and AD Tg + GEF (50 or $100 \mathrm{mg} / \mathrm{kg}$, p.o., $\mathrm{n}=8$ ) groups. The six-month-old AD Tg mice were treated with saline or gintonin (50 or $100 \mathrm{mg} / \mathrm{kg}$, orally) 3 times a week for 3 months (17). All analyses were performed when the mice were 9 months old. The number of mice which died during 9 months old was $0,1,2$ and 1 in wild-type, AD Tg treated with saline, AD $\mathrm{Tg}+\mathrm{GEF}(50 \mathrm{mg} / \mathrm{kg})$, and AD Tg + GEF (100 mg/kg) group, respectively. All experimental procedures were conducted in a blinded manner in accordance with the Guide for the Care and Use of Laboratory Animals of the National Institutes of Health. The protocol was approved by the Institutional Animal Care and Use Committees of Kangwon (Permit no. 13-156) and Konkuk (Permit no. 14-956) Universities. Sodium pentobarbital $(50 \mathrm{mg} / \mathrm{kg}$, i.p.) was used for mouse anesthesia and mice were sacrificed by cervical dislocation when they did not respond to paw stimuli.

Brain and section preparation. The brains and sections were prepared as previously described with slight modifications (19). Mice from WT $(n=4), \operatorname{AD~Tg}(n=4)$, and AD Tg +50 or $100 \mathrm{mg} / \mathrm{kg} \mathrm{GEF} \mathrm{(n=4,} \mathrm{each)} \mathrm{groups} \mathrm{were} \mathrm{sacrificed} \mathrm{using}$ 
diethyl ether and were perfused intracardially with $0.9 \%$ saline and fixative with $4 \%$ paraformaldehyde in $0.1 \mathrm{M}$ phosphate buffer ( $\mathrm{pH}$ 7.4). The brains were fixed with the same fixative overnight at $4^{\circ} \mathrm{C}$. The brains were serially cryoprotected in 10, 20 and $30 \%$ sucrose in phosphate-buffered saline for $48 \mathrm{~h}$ at $4{ }^{\circ} \mathrm{C}$ and coronally sliced into $30-\mu \mathrm{m}$-thick sections with a freezing microtome (model: CM3050S, Leica Biosystems) for immunohistochemical and immunofluorescent staining.

Quantification of BBB permeability. The level of BBB disruption was detected by the quantitative measurement of Evans blue content as previously described (21). Briefly, 2\% sterilized Evans blue dye (Sigma-Aldrich; Merck KGaA) solution was intravenously injected at a dose of $4.0 \mathrm{ml} / \mathrm{kg}$ per mouse in WT $(\mathrm{n}=4), \operatorname{AD} \mathrm{Tg}(\mathrm{n}=4)$, and AD Tg +50 or $100 \mathrm{mg} / \mathrm{kg}$ GEF ( $n=4$, each) groups. Thirty minutes after the injection, the mice were perfused with saline to remove Evans blue dye from the vascular system. The brains were immediately removed, sliced into $2 \mathrm{~mm}$ thickness using brain matrix (Cell Point Scientific), and fixed with $4 \%$ paraformaldehyde solution. Cryosections of $30 \mu \mathrm{m}$ thickness were prepared and immunofluorescent staining of PECAM-1 was performed as previously described (21). Staining with Evans blue and immunofluorescent staining of PECAM-1 were used to detect albumin extravasation (BBB permeability) and BBB disruption, respectively.

Quantitative real-time PCR analysis. For real-time PCR analysis, the hippocampus from the right hemisphere of each mouse groups were used after harvest and the total RNA was extracted using TRIsure reagent according to the manufacturer's instructions (Bioline). Real-time PCR analysis was performed as previously described (21). Briefly, the hippocampus were reacted with the following primer sets: ICAM-1, 5'-TGC GTT TTG GAG CTA GCG GAC CA-3' and 5'-CGA GGA CCA TAC AGC ACG TGC AG-3'; VCAM-1, 5'-CCT CAC TTG CAG CAC TAC GGG CT-3' and 5'-TTT TCC AAT ATC CTC AAT GAC GGG-3'; claudin-3, 5'-CTG GGA GGG CCT GTG GAT GAA CT-3' and 5'-TCG CGG CGC AGA ATA GAG GAT-3'; and glyceraldehyde 3-phosphate dehydrogenase, 5'-AGG TCA TCC CAG AGC TGA ACG-3' and 5'-CAC CCT GTT GCT GTA GCC GTA T-3'. All real-time PCR experiments were performed at least three times, and the expression of each gene was normalized to that of glyceraldehyde 3-phosphate dehydrogenase.

Immunohistochemical and immunofluorescence evaluations. Immunohistochemical staining was performed as previously described (21). Briefly, the free-floating sections ( $n=4$ per brain) of each mouse from WT ( $=4), \operatorname{AD~Tg~}(n=4)$, and AD Tg + 50 or $100 \mathrm{mg} / \mathrm{kg}$ GEF ( $\mathrm{n}=4$, each) groups were reacted with rabbit anti- $\beta$-amyloid antibody (1:200; Invitrogen; Thermo Fisher Scientific, Inc.) and biotinylated secondary antibody (1:200; Vector Laboratories), and were visualized using an avidin-biotinylated horseradish peroxidase complex Elite ${ }^{\circledR}$ kit (Vector Laboratories) and 3,3'-diaminobenzidine. The sections were analyzed using a DP70 image analysis system (Olympus Co.). $\beta$-amyloid plaque deposition in the parietal cortex and hippocampus were quantified using ImageJ (http://rsb.info. nih.gov/ij/). The plaque deposition was also quantified as previously described (17). The images of $\beta$-amyloid-stained sections were compared to 6-grade plaque photographic reference panels (17) and assigned a numerical grade ranging from 0 to 6 . Immunofluorescence staining was performed as previously described (21). Briefly, the floating brain sections from all mice ( $n=3$ per brain) were incubated with rabbit anti-PECAM-1 (1:500; Santa Cruz Biotechnology, Inc.), mouse anti-occludin, claudin-5, and ZO-1 (1:500; Invitrogen; Thermo Fisher Scientific, Inc.) antibodies and secondary antibody (1:200; Vector Laboratories), and examined with a confocal imaging system (LSM 5 PASCAL; Carl Zeiss). The captured color images were converted to 8-bit gray-scale images and the specific signals were detected from a nonspecific background by a threshold value that was set between 25 and 30 . The area densities of immunofluorescent positive area were calculated as the proportion of pixels having higher fluorescent intensities than threshold value.

Statistical analyses. All statistical analyses were performed using SPSS package version 21.0 (SPSS Inc.) for Windows. Multiple comparisons were performed using one-way ANOVA with Tukey's post hoc test for the data in Figs. 2, 3 and S1 and non-parametric Kruskal Wallis test with post hoc test for the data in Fig. 1. All data are presented as the mean \pm SEM, and the statistical difference was detected at a $5 \%$ level unless otherwise indicated.

\section{Results}

Effects of GEF on $\beta$-amyloid plaque deposition in the brain of AD Tg mice. The six-month-old AD Tg mice were first treated with saline or GEF (50 or $100 \mathrm{mg} / \mathrm{kg}$, p.o.) 3 times a week for 3 months. Next, to evaluate whether long-term oral administration of GEF reduced brain $\beta$-amyloid depositions, we first examined the plaque burdens in the parietal cortex and hippocampus of AD Tg mice by immunohistochemical analyses. We observed that the number of $\beta$-amyloid plaques and grade of $\beta$-amyloid plaque depositions were significantly higher in AD Tg mice than that in wild-type mice (Fig. 1A, B, E and F), whereas the depositions were significantly inhibited by long-term oral administration of GEF with dose-dependent manner (Fig. 1C, D, E and F). These results indicated that GEF has inhibitory effects against $\beta$-amyloid plaque depositions in AD Tg mice, which is well consistent with previous reports $(17,18)$.

Effects of GEF on Evans blue permeability in AD Tg mice. Since the amyloid plaque accumulations in AD brain are associated with brain blood vessel disruptions including BBB (12), we further examined whether brain microvascular permeability was increased in AD Tg mice and whether long-term oral administration of GEF can attenuate the increased brain microvascular leakage. We used Evans blue as a marker of BBB integrity (22). In the present study, we found that compared to cortex and hippocampus of WT mice (Fig. 2A, D and G), the level of Evans blue staining was significantly higher around the PECAM-1 positive endothelial cells in the cortex and hippocampus of $\mathrm{AD} \mathrm{Tg}$ mice (Fig. 2B, E and G), whereas long-term oral administration of GEF significantly inhibited the increased magnitude of Evans blue staining and amount of the dye in cortex and hippocampus 


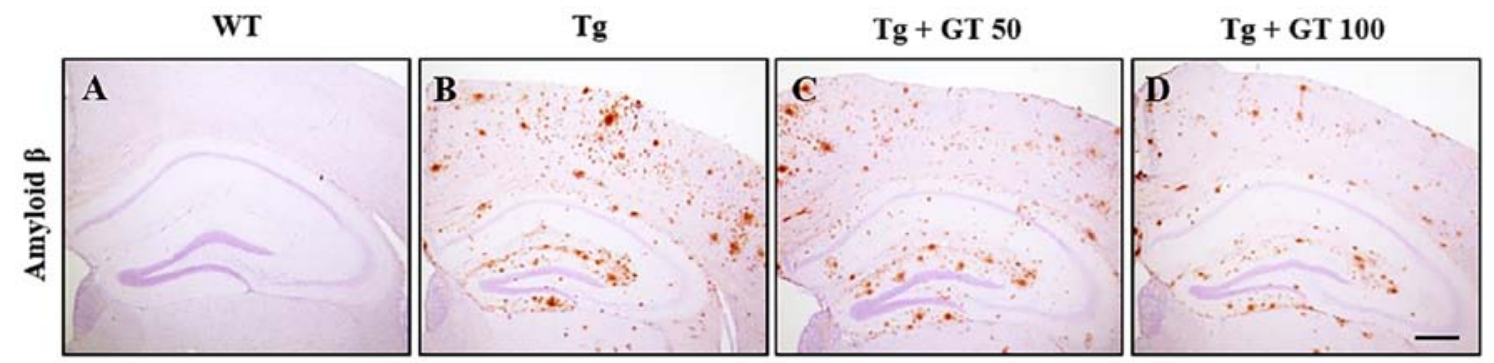

$\mathbf{E}$

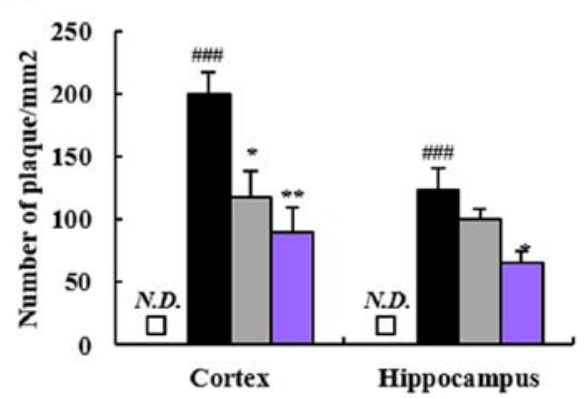

F

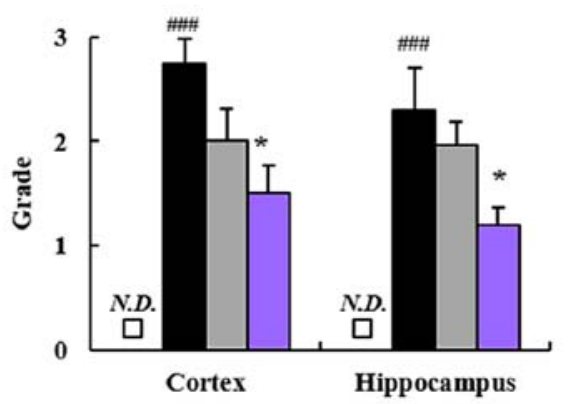

$\square$ WT

- $\mathbf{T g}$

$\square \mathrm{Tg}+\mathrm{GT} 50$

Tg + GT 100

Figure 1. GEF inhibited $\beta$-amyloid depositions in the brain of AD Tg mice. (A-C) A period of three months after 50 or $100 \mathrm{mg} / \mathrm{kg}$ GEF treatment (mice were 9 months old), brain sections ( $\mathrm{n}=3$ per brain) from WT ( $=4)$, AD Tg ( $=4)$, and AD Tg +50 or $100 \mathrm{mg} / \mathrm{kg} \mathrm{GEF}$ ( $\mathrm{n}=4$, each) groups of mice were immunostained using antibody for $\beta$-amyloid ( $\mathrm{D}$ and $\mathrm{E}$ ). The number of $\beta$-amyloid plaques in the parietal cortex and hippocampus were estimated using (D) ImageJ and ( $\mathrm{E}$ and F) numerical grades were assigned to the plaque deposition levels of 50 or $100 \mathrm{mg} / \mathrm{kg}$ GEF treatment. Scale bar, $100 \mu \mathrm{m}$. ${ }^{\# \# ~} \mathrm{P}<0.001 \mathrm{vs}$. WT mice; ${ }^{*} \mathrm{P}<0.05$ and ${ }^{* *} \mathrm{P}<0.01$ vs. AD Tg mice alone. GEF, gintonin-enriched fraction; AD, Alzheimer's disease; WT, wild-type; ND, not determined.
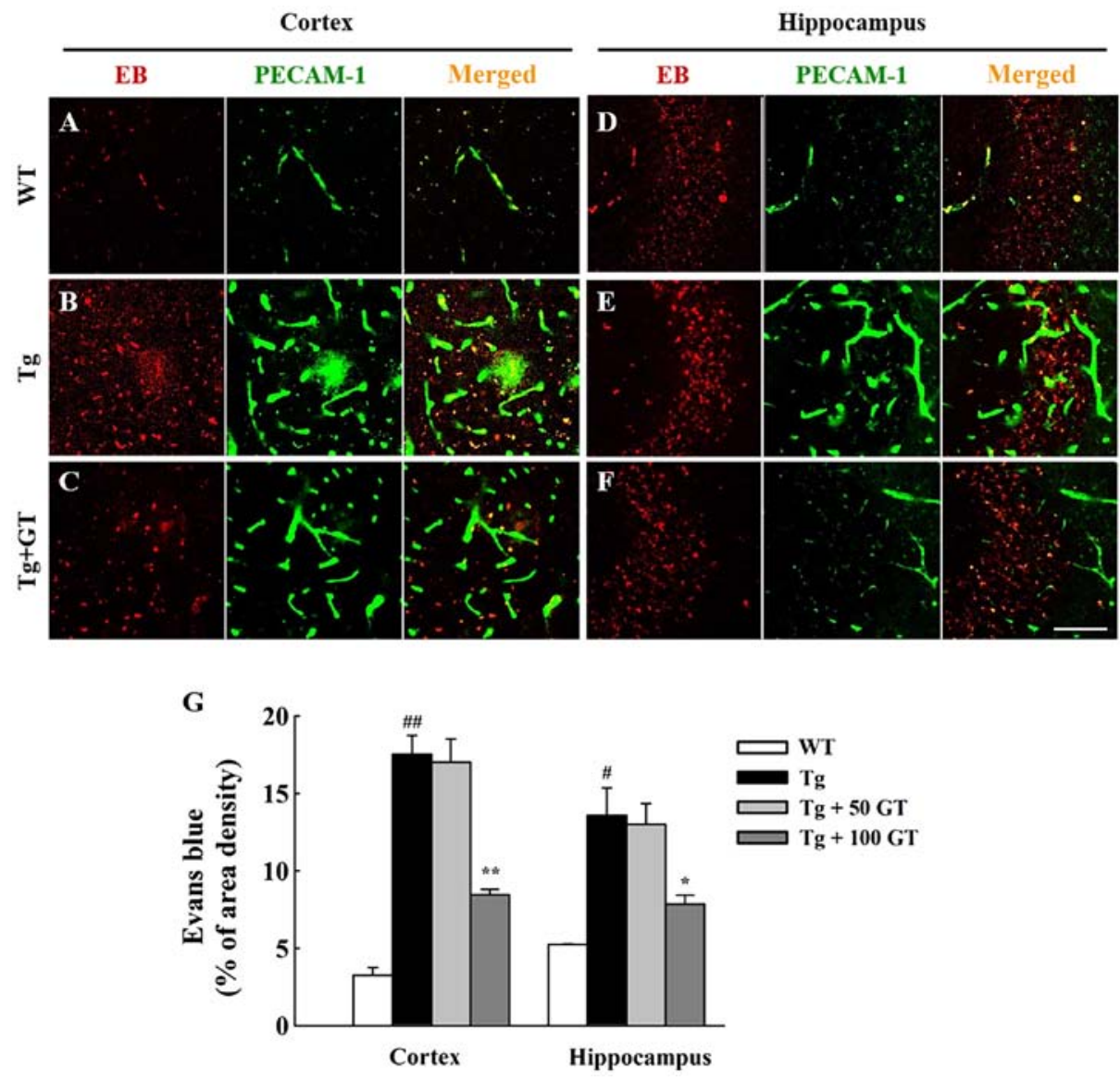

Figure 2. GEF reduced BBB permeability in the brain of AD mice. Three months after GEF treatment (mice were 9 months old), sterilized Evans blue solution was intravenously injected to mice of WT $(n=4), \operatorname{AD~Tg}(n=4)$, and AD Tg + GEF (n=4) groups. A period of 30 min after injection, brain sections (n=3 per brain) were prepared, immunofluorescence staining was performed using an antibody against PECAM-1. The representative pictures of Evans blue (left panel of A-C and D-F) and PECAM-1 (middle panel of A-C and D-F) were captured and quantified with (G) two different dosages of GEF. Scale bar, $50 \mu \mathrm{m}$. ${ }^{*} \mathrm{P}<0.05$ and ${ }^{\# \#} \mathrm{P}<0.01$ vs. WT mice; ${ }^{*} \mathrm{P}<0.05$ and ${ }^{* * *} \mathrm{P}<0.01$ vs. AD Tg mice alone. GEF, gintonin-enriched fraction; BBB, brain-blood barrier; AD, Alzheimer's disease; WT, wild-type; ZO-1, zonula occludens-1. 
A

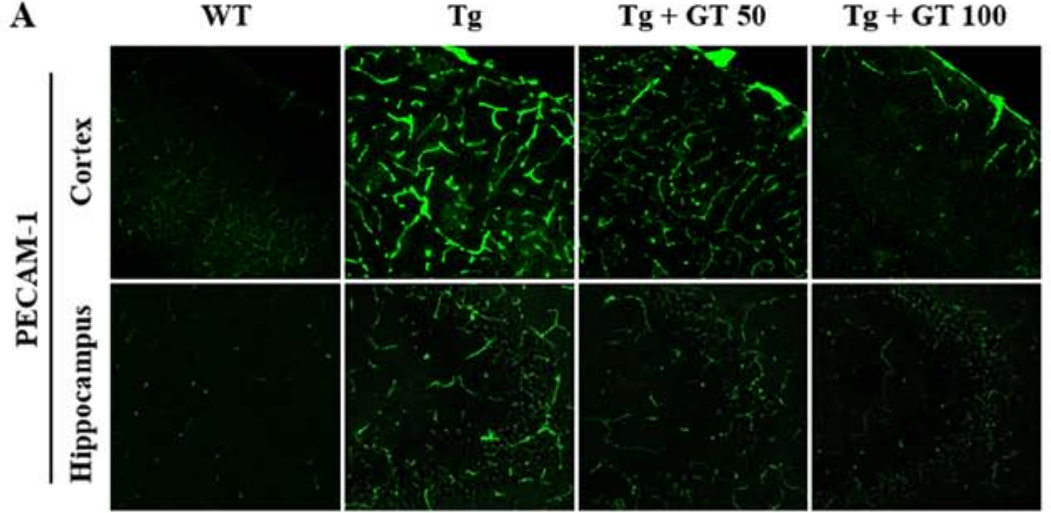

C

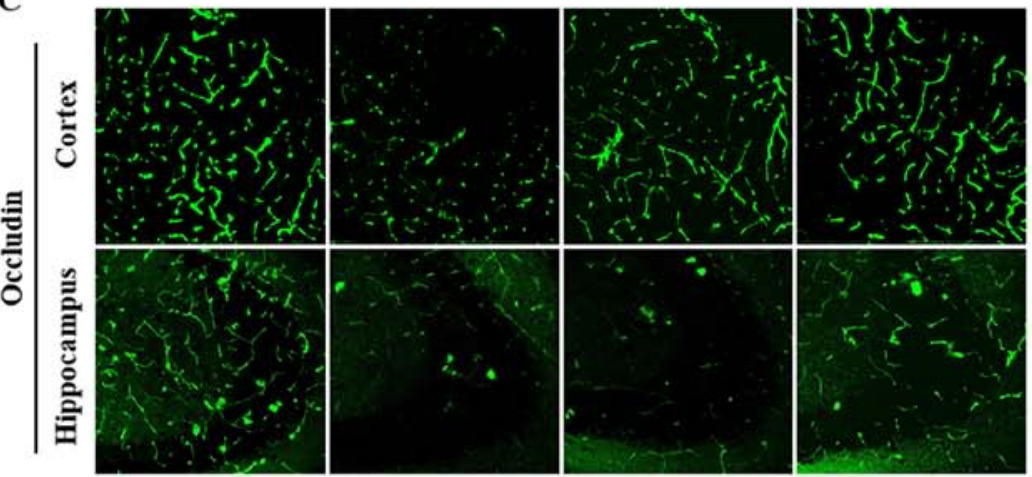

E

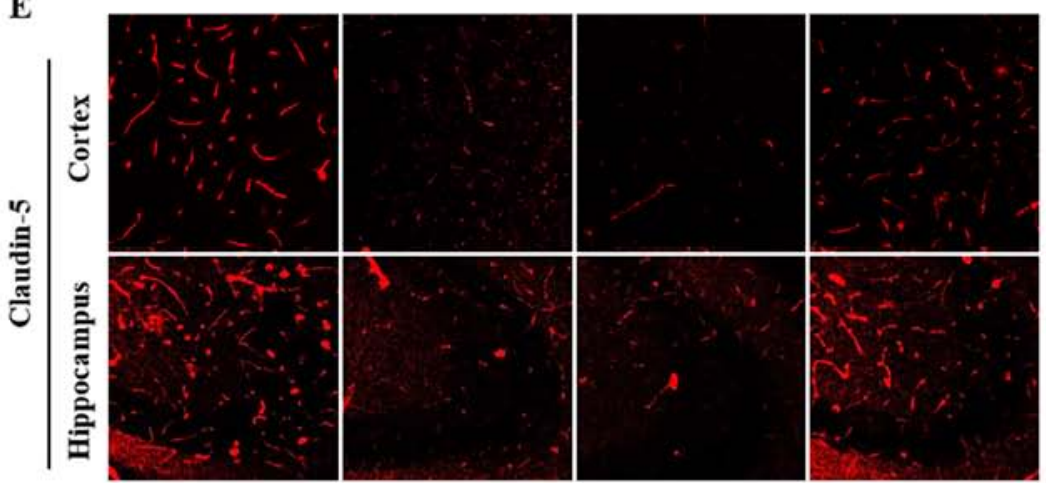

G

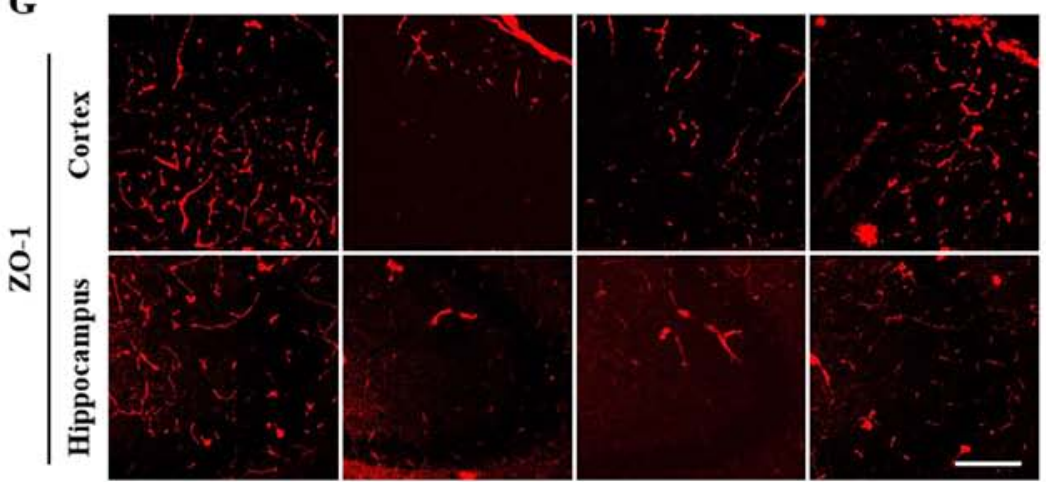

B

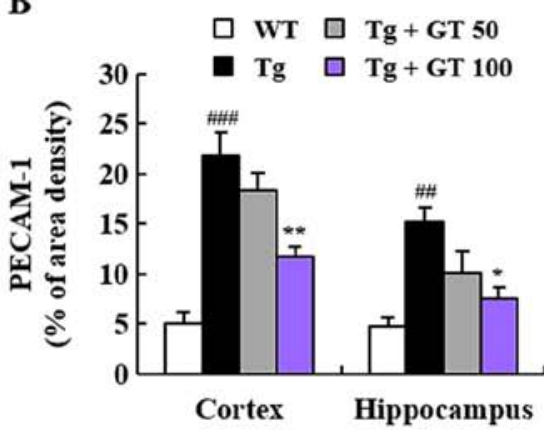

D

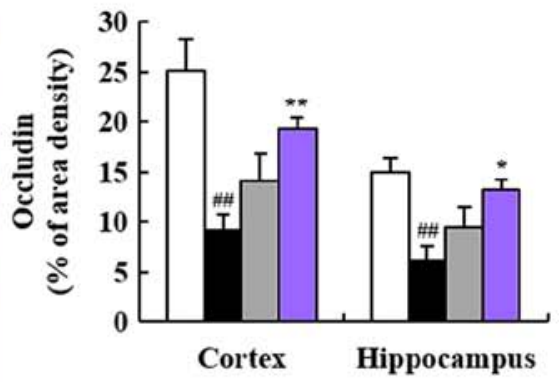

F

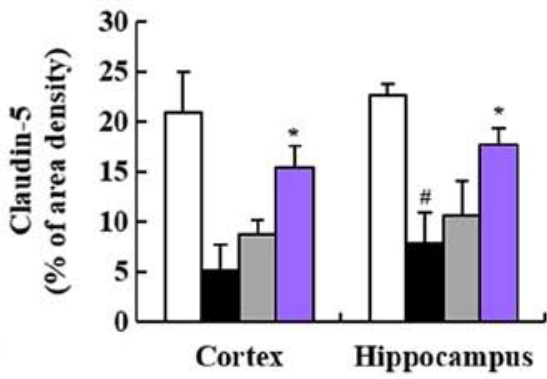

H

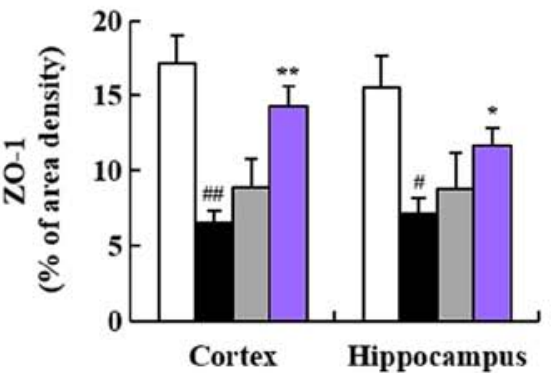

Figure 3. GEF maintained the BBB integrity in the brain of AD mice. A period of 3 months after GEF treatment (mice were 9 months old), brain sections ( $\mathrm{n}=3$ per brain) including the parietal cortex and hippocampus (CA3) from WT ( $=4)$, AD Tg ( $=4)$, and AD Tg + 50 or $100 \mathrm{mg} / \mathrm{kg}$ GEF ( $=4$, each) groups were analyzed by the immunofluorescence staining using an antibodies against (A) PECAM-1, (C) occludin, (E) claudin-5 and (G) ZO-1 and quantified (B, D, F and H) Scale bar, $100 \mu \mathrm{m} .{ }^{\#} \mathrm{P}<0.05$ and ${ }^{\# \#} \mathrm{P}<0.01$ vs. WT mice; ${ }^{\# \# \#} \mathrm{P}<0.001$ vs. WT mice; ${ }^{*} \mathrm{P}<0.05$ and ${ }^{* *} \mathrm{P}<0.01$ vs. AD Tg mice alone. GEF, gintonin-enriched fraction; $\mathrm{BBB}$, brain-blood barrier; AD, Alzheimer's disease; WT, wild-type; ZO-1, zonula occludens-1.

with dose-dependent manner (Fig. 2C, F and G). The GEF effect corroborated with the alteration in PECAM-1 (Figs. 2 and 3), indicating that long-term administration of GEF might assist in the maintenance of BBB integrity in the AD animal model.
Effects of GEF on maintenance of BBB integrity in $A D T g$ mice. Since long-term administration of GEF reduced the leakage of brain microvessels in $\mathrm{AD}$ as observed in the Evans blue staining (Fig. 2), we further evaluated the effects 
of GEF on the disruptions of BBB components in cortex and hippocampus that form the BBB. We first compared PECAM-1 (CD31) protein expression in AD Tg mice with that in WT mice as an indicator of BBB disruption $(23,24)$. We found that compared to WT mice, the immunofluorescence level of PECAM-1 was higher in the cortex and hippocampus of AD Tg mice (Fig. 3A and B), although this elevated expression was significantly inhibited by long-term oral treatment with GEF with dose-dependent manner (Fig. 3A and B). Since BBB disruption is also related to the alterations in the levels of intercellular tight junction molecules $(25,26)$, we also investigated whether GEF had further protective effects on the protein expression of junctional molecules using immunofluorescence staining. The expression levels of occludin, claudin-5, and $\mathrm{ZO}-1$, the main junctional proteins, was downregulated in the cortex and hippocampus of $\mathrm{AD} \mathrm{Tg}$ mice as compared to WT mice (Fig. 3C-H). However, long-term oral GEF treatment significantly prevented this downregulation (Fig. 3C-H). We additionally investigated whether GEF had further protective effects on the gene expression of the representative molecules using quantitative real-time PCR. The mRNA expression of claudin-3, a junctional protein, was downregulated in the hippocampus of AD Tg mice as compared to WT mice, whereas long-term GEF treatment prevented this downregulation (Fig. S1A). The mRNA expression of endothelial ICAM-1 and VCAM-1, two intercellular adherens junction proteins, were upregulated in the hippocampus of $\mathrm{AD} \mathrm{Tg}$ mice as compared to WT mice, whereas GEF treatment attenuated the upregulation of ICAM-1 and VCAM-1 expression (Fig. S1B and C). These results suggest that GEF might exert protective effects on BBB disruption induced by $\beta$-amyloid plaque depositions in $\mathrm{AD} \mathrm{Tg}$ mice through regulation of the differential gene or protein expression of adherens and junctional molecules.

\section{Discussion}

The endothelium is located inside most of the blood vessel, including the brain microvessels which consists of a monolayer of endothelial cells. Inter-endothelial connections, such as adherens junctions and tight junctions contain a variety of junctional proteins (27). These inter-connecting proteins in the endothelium contribute to the integrity of the BBB. Considering that in vitro deposition of $\beta$-amyloid leads to BBB disintegration and $\mathrm{BBB}$ dysfunctions in $\mathrm{AD}$ resulting in an alteration in BBB permeability $(9,28)$, we investigated whether long-term treatment via oral GEF could alter $\beta$-amyloid plaque deposition, leakage of brain microvessels, and expression of intercellular tight junction proteins in AD Tg mice.

In the present study, we showed that accumulations of amyloid plaques in the brain are closely associated with disruption of brain microvascular integrity. First, we found that GEF administration attenuated $\beta$-amyloid plaque accumulations in cortex and hippocampus, of which is comparable with donepezil used as a positive drug in previous study (17). Next, we showed that GEF-mediated attenuation of $\beta$-amyloid plaque accumulations helped in restoring the brain microvascular integrity as observed by Evans blue test and immunohistochemical studies with endothelial junctional proteins such as PECAM-1, occludin, claudin-5 and ZO-1 (Figs. 1-3). In the
Evans blue test, we observed that long-term administration of GEF restored BBB permeability near to wild-type level with $100 \mathrm{mg} / \mathrm{kg}$ GEF (Fig. 2). Regarding the endothelial adherens proteins, long-term GEF treatment reduced the expression of PECAM-1, ICAM-1, and VCAM-1, of which expressions are increased by $\beta$-amyloids $(11,28)$. In addition, $\beta$-amyloid induced inflammation in the brain led to activation of microglia, increased ICAM-1 expression, and disruption of BBB integrity (28). GEF, however, attenuated the increased gene expression of claudin-3, ICAM-1, and VCAM-1 near to the wild-type level in the hippocampus of AD Tg mice (Fig. S1). In addition, $\beta$-amyloid accumulations affected PECAM-1, occludin, claudin-5, and ZO-1 expression level, resulting in disruption of BBB integrity (28). GEF, however, also attenuated the increased expression of PECAM-1 near to the wild-type level and also restored the decreased occludin, claudin-5 and ZO-1 protein expression level in the cortex and hippocampus of AD Tg mice near to the wild-type (Fig. 3), demonstrating that GEF-mediated attenuation of amyloid plaque accumulations was closely related to brain microvascular integrity.

Occludin, claudin-5, and ZO-1, members of junctional protein, participates in the formation of tight junctions among the brain microvascular endothelial cells. Although the selective loss of occludin, claudin-5, and ZO-1 from BBB tight junctions is demonstrated under different pathological conditions, such as experimental autoimmune encephalomyelitis and human glioblastoma multiforme $(29,30)$, alteration in claudin-5 expression by in vivo $\beta$-amyloid plaque accumulation is not currently unknown. In the present study, occludin, claudin-5, and ZO-1, expression was reduced in the cortex and hippocampus of $\mathrm{AD} \mathrm{Tg}$ mice, whereas oral administration of GEF increased the expression of occludin, claudin-5, and ZO-1. These findings supported that claudin-5, occludin, and ZO-1 might also be involved in the pathology of AD by affecting tight junction integrity and GEF treatment could restore the claudin-5, occludin and ZO-1 level (Fig. 3).

It is noteworthy to consider the molecular mechanisms which are involved in the beneficial effects of GEF on the integrity of brain microvascular endothelium in $\mathrm{AD} \mathrm{Tg}$ mice. First, LPA plays a key role in angiogenesis via LPARs (8). In a previous study, we have shown that gintonin stimulated proliferation, migration, and tube formation in human umbilical vein endothelial cells via LPA $1 / 3$ receptor activations (31). GEF also increased the release of vascular endothelial growth factors (VEGF) from human umbilical vein endothelial cells and astrocytes through LPAR membrane signaling $(31,32)$. VEGF is involved in maintaining the integrity of BBB and its ability to significantly prevent $\beta$-amyloid-induced endothelial cell apoptosis $(33,34)$. Thus, the GEF-mediated release of VEGF from the brain microvascular endothelial cells and astrocytes might contribute to the restoration of AD-induced changes in the blood vessels and junctional proteins of the brain. Second, $\beta$-amyloid, which induces neuroinflammation and oxidative stress in the brain microvessels can disrupt the integrity of the brain microvascular endothelium by modifying the inter-junctional protein expression and microvascular permeability $(35,36)$. GEF-mediated anti-neuroinflammatory and anti-oxidative effects via LPA receptors might protect the brain microvascular endothelium from disintegrations following $\beta$-amyloid plaque deposition. 
In fact, we showed that both in vitro treatment of RAW 264.7 cells with gintonin and in vivo administration of gintonin to AD Tg and Parkinson's disease and Huntington's disease model mice were found to inhibit microglial activation and formation of reactive oxygen species (17,37-41). Thus, gintonin-mediated VEGF release in brain microvessels and astrocytes and gintonin-mediated anti-neuroinflammatory and anti-oxidant effects might contribute to the brain microvascular integrity in $\mathrm{AD} \mathrm{Tg}$ mice.

In addition, it is worthwhile to note the differential regulations of gintonin on the brain microvascular functions. In a previous report, we have shown that acute intravenous administration of gintonin opened BBB transiently and increased BBB permeability through LPA1/3 receptor signaling pathways (42). We further showed that gintonin itself could enter the brain and bind to the neuronal cells (42). Thus, previous and present studies demonstrate that gintonin could exert differential regulatory functions on brain $\mathrm{BBB}$ that might be dependent on the route and duration of its administration. This hypothesis is supported by the fact that after acute oral administration of the same dose of gintonin that was used for the long-term treatment in the AD mouse model, we could not observe any effect of gintonin on the brain permeability through transient BBB opening (data not shown). Thus, although it is likely that acute intravenous but not oral administration of gintonin opens BBB transiently, further studies are required to elucidate the molecular mechanisms of the differential effects of gintonin on brain $\mathrm{BBB}$ regulation.

Taken together, the present study demonstrated that $\beta$-amyloid plaque depositions might induce changes in the junctional protein expressions eventually disrupting the integrity of BBB, which is linked to the increased BBB permeability. Long-term oral administration of GEF to AD Tg mice, however, exerted protective effects against brain microvascular disruptions as well as $\beta$-amyloid plaque depositions (Figs. 1-3). The present study is well-consistent with previous report that long-term oral administration of GEF to Parkinson's disease animal model mice also protect from BBB disruptions induced by 1-Methyl-4-phenyl-1,2,3,6-tetrahydropyridine (40). Thus, the present study might further expand the previous molecular knowledges on the gintonin-mediated anti-AD effects.

In summary, the present study showed that long-term oral treatment of GEF attenuated the disruptions of BBB permeability and endothelial inter-junctional proteins observed in the brain microvessels of AD Tg mice. Taken together, the present study demonstrated a possibility that gintonin-mediated anti-AD effects might be achieved through mitigations of the microvascular damages and attenuations of amyloid plaque accumulation in the brain.

\section{Acknowledgements}

Not applicable.

\section{Funding}

This research was supported by the Basic Science Research Program and Brain Research Program through the National Research Foundation of Korea (NRF) funded by the Ministry of Science and ICT (grant nos. NRF-2012R1A1A2008505,
NRF-2016M3C7A1905074, NRF-2016M3C7A1913845, NRF-2017R1A2A2A05069493 and NRF-2020R1F1A1058460). The current study was supported by Konkuk University Researcher Fund in 2019.

\section{Availability of data and materials}

The datasets used and/or analyzed during the current study are available from the corresponding author on reasonable request.

\section{Authors' contributions}

MJ, SHC, IHC, and SYN conceived and designed the study and drafted the manuscript. MJ, SHC, JHC, and JO performed the experiments. MJ, SHC, JHC, RML, NEL, YJC, JO and SYN performed the investigation. RML, NEL and YJC analyzed data and prepared figures. HR and HCK provided the resources. HR, HCK and JO supervised the research. All authors read and approved the final version of the manuscript.

\section{Ethics approval and consent to participate}

All experimental procedures were conducted in a blinded manner in accordance with the Guide for the Care and Use of Laboratory Animals of the National Institutes of Health. The protocol was approved by the Institutional Animal Care and Use Committees of Kangwon (no. 13-156) and Konkuk (no. 14-956) Universities.

\section{Patient consent for publication}

Not applicable.

\section{Competing interests}

The authors declare that they have no competing interests.

\section{References}

1. Yung YC, Stoddard NC, Mirendil H and Chun J: Lysophosphatidic Acid signaling in the nervous system. Neuron 85: 669-682, 2015.

2. Choi JW, Herr DR, Noguchi K, Yung YC, Lee CW, Mutoh T, Lin ME, Teo ST, Park KE, Mosley AN, et al: LPA receptors: Subtypes and biological actions. Annu Rev Pharmacol Toxicol 50: 157-186, 2010.

3. Hecht JH, Weiner JA, Post SR and Chun J: Ventricular zone gene-1 (vzg-1) encodes a lysophosphatidic acid receptor expressed in neurogenic regions of the developing cerebral cortex. J Cell Biol 135: 1071-1083, 1996.

4. Ladrón de Guevara-Miranda D, Moreno-Fernández RD, Gil-Rodríguez S, Rosell-Valle C, Estivill-Torrús G, Serrano A, Pavón FJ, Rodríguez de Fonseca F, Santín LJ and Castilla-Ortega E: Lysophosphatidic acid-induced increase in adult hippocampal neurogenesis facilitates the forgetting of cocaine-contextual memory. Addict Biol 24: 458-470, 2018).

5. Lin C-I, Chen C-N, Lin P-W, Chang K-J, Hsieh F-J and Lee H: Lysophosphatidic acid regulates inflammation-related genes in human endothelial cells through LPA1 and LPA3. Biochem Biophys Res Commun 363: 1001-1008, 2007.

6. Ren Y, Guo L, Tang X, Apparsundaram S, Kitson C, Deguzman J, Fuentes ME, Coyle L, Majmudar R, Allard J, et al: Comparing the differential effects of LPA on the barrier function of human pulmonary endothelial cells. Microvasc Res 85: 59-67, 2013.

7. Baker DL, Desiderio DM, Miller DD, Tolley B and Tigyi GJ: Direct quantitative analysis of lysophosphatidic acid molecular species by stable isotope dilution electrospray ionization liquid chromatography-mass spectrometry. Anal Biochem 292: 287-295, 2001. 
8. Rivera-Lopez CM, Tucker AL and Lynch KR: Lysophosphatidic acid (LPA) and angiogenesis. Angiogenesis 11: 301-310, 2008.

9. Rosenberg GA: Blood-brain barrier permeability in aging and Alzheimer's disease. J Prev Alzheimers Dis 1: 138-139, 2014

10. van de Haar HJ, Burgmans S, Jansen JF, van Osch MJ, van Buchem MA, Muller M, Hofman PA, Verhey FR and Backes WH: Blood-brain barrier leakage in patients with early Alzheimer disease. Radiology 281: 527-535, 2016.

11. Zenaro E, Piacentino G and Constantin G: The blood-brain barrier in Alzheimer's disease. Neurobiol Dis 107: 41-56, 2017.

12. Chakraborty A, de Wit NM, van derFlier WM and de Vries HE: The blood brain barrier in Alzheimer's disease. Vascul Pharmacol 89: 12-18, 2017

13. Hwang SH, Shin TJ, Choi SH, Cho HJ, Lee BH, Pyo MK, Lee JH, Kang J, Kim HJ, Park CW, et al: Gintonin, newly identified compounds from ginseng, is novel lysophosphatidic acids-protein complexes and activates $\mathrm{G}$ protein-coupled lysophosphatidic acid receptors with high affinity. Mol Cells 33: 151-162, 2012.

14. Choi SH, Jung SW, Lee BH, Kim HJ, Hwang SH, Kim HK and Nah SY: Ginseng pharmacology: A new paradigm based on gintonin-lysophosphatidic acid receptor interactions. Front Pharmacol 6: 245, 2015

15. Park H, Kim S, Rhee J, Kim HJ, Han JS, Nah SY and Chung C: Synaptic enhancement induced by gintonin via lysophosphatidic acid receptor activation in central synapses. J Neurophysiol 113: 1493-1500, 2015

16. Kim S, Kim MS, Park K, Kim HJ, Jung SW, Nah SY, Han JS and Chung C: Hippocampus-dependent cognitive enhancement induced by systemic gintonin administration. J Ginseng Res 40 : 55-61, 2016.

17. Hwang SH, Shin EJ, Shin TJ, Lee BH, Choi SH, Kang J, Kim HJ, Kwon SH, Jang CG, Lee JH, et al: Gintonin, a ginseng-derived lysophosphatidic acid receptor ligand, attenuates Alzheimer's disease-related neuropathies: Involvement of non-amyloidogenic processing. J Alzheimers Dis 31: 207-223, 2012.

18. Kim HJ, Shin EJ, Lee BH, Choi SH, Jung SW, Cho IH, Hwang SH, Kim JY, Han JS, Chung C, et al: Oral Administration of gintonin attenuates cholinergic impairments by scopolamine, amyloid- $\beta$ protein, and mouse model of Alzheimer's disease. Mol Cells 38 796-805, 2015

19. Choi JH, Jang M, Nah SY, Oh S and Cho IH: Multitarget effects of Korean Red Ginseng in animal model of Parkinson's disease: Antiapoptosis, antioxidant, antiinflammation, and maintenance of blood-brain barrier integrity. J Ginseng Res 42: 379-388, 2018

20. Cho HJ, Choi SH, Kim HJ, Lee BH, Rhim H, Kim HC, Hwang SH and Nah SY: Bioactive lipids in gintonin-enriched fraction from ginseng. J Ginseng Res 43: 209-217, 2019.

21. Lee MJ, Jang M, Choi J, Chang BS, Kim DY, Kim SH, Kwak YS, Oh S, Lee JH, Chang BJ, et al: Korean red ginseng and ginsenoside-Rb1/-Rg1 alleviate experimental autoimmune encephalomyelitis by suppressing Th1 and Th17 cells and upregulating regulatory T cells. Mol Neurobiol 53: 1977-2002, 2016.

22. Saunders NR, Dziegielewska KM, Møllgård K and Habgood MD: Markers for blood-brain barrier integrity: How appropriate is Evans blue in the twenty-first century and what are the alternatives? Front Neurosci 9: 385, 2015.

23. Flynn KM, Michaud M, Canosa S and Madri JA: CD44 regulates vascular endothelial barrier integrity via a PECAM-1 dependent mechanism. Angiogenesis 16: 689-705, 2013.

24. Kalinowska A and Losy J: PECAM-1, a key player in neuroinflammation. Eur J Neurol 13: 1284-1290, 2006.

25. Al-Obaidi MM and Desa MN: Mechanisms of blood brain barrier disruption by different types of bacteria, and bacterial-host interactions facilitate the bacterial pathogen invading the brain. Cell Mol Neurobiol 38: 1349-1368, 2018

26. Almutairi MM, Gong C, Xu YG, Chang Y and Shi H: Factors controlling permeability of the blood-brain barrier. Cell Mol Life Sci 73: 57-77, 2016.

27. Wallez Y and Huber P: Endothelial adherens and tight junctions in vascular homeostasis, inflammation and angiogenesis. Biochim Biophys Acta 1778: 794-809, 2008.
28. Marco S and Skaper SD: Amyloid beta-peptide1-42 alters tight junction protein distribution and expression in brain microvessel endothelial cells. Neurosci Lett 401: 219-224, 2006.

29. Liu Z, Liu J, Wang S, Liu S and Zhao Y: Neuronal uptake of serum albumin is associated with neuron damage during the development of epilepsy. Exp Ther Med 12: 695-701, 2016.

30. Wolburg H, Wolburg-Buchholz K, Kraus J, Rascher-Eggstein G, Liebner S, Hamm S, Duffner F, Grote EH, Risau W and Engelhardt B: Localization of claudin-3 in tight junctions of the blood-brain barrier is selectively lost during experimental autoimmune encephalomyelitis and human glioblastoma multiforme. Acta Neuropathol 105: 586-592, 2003.

31. Hwang SH, Lee BH, Choi SH, Kim HJ, Won KJ, Lee HM, Rhim H, Kim H-C and Nah SY: Effects of gintonin on the proliferation, migration, and tube formation of human umbilical-vein endothelial cells: Involvement of lysophosphatidic-acid receptors and vascular-endothelial-growth-factor signaling. J Ginseng Res 40: 325-333, 2016.

32. Choi SH, Kim HJ, Cho HJ, Park SD, Lee NE, Hwang SH, Rhim H, Kim HC, Cho IH and Nah SY: Gintonin-mediated release of astrocytic vascular endothelial growth factor protects cortical astrocytes from hypoxia-induced cell damages. J Ginseng Res 43: 305-311, 2019.

33. Religa P, Cao R, Religa D, Xue Y, Bogdanovic N, Westaway D, Marti HH, Winblad B and Cao Y: VEGF significantly restores impaired memory behavior in Alzheimer's mice by improvement of vascular survival. Sci Rep 3: 2053, 2013.

34. Shen F, Walker EJ, Jiang L, Degos V, Li J, Sun B, Heriyanto F, Young WL and Su H: Coexpression of angiopoietin-1 with VEGF increases the structural integrity of the blood-brain barrier and reduces atrophy volume. J Cereb Blood Flow Metab 31: 2343-2351, 2011.

35. Balducci C and Forloni G: Novel targets in Alzheimer's disease: A special focus on microglia. Pharmacol Res 130: 402-413, 2018

36. Cai Z, Zhao B and Ratka A: Oxidative stress and $\beta$-amyloid protein in Alzheimer's disease. Neuromolecular Med 13: 223-250, 2011.

37. Saba E, Jeon BR, Jeong DH, Lee K, Goo YK, Kwak D, Kim S, Roh SS, Kim SD, Nah SY, et al: A novel Korean red ginseng compound gintonin inhibited inflammation by MAPK and NF- $\mathrm{kB}$ pathways and recovered the levels of mir-34a and mir-93 in RAW 264.7 cells. Evid Based Complement Alternat Med 2015: $624132,2015$.

38. Jo MG, Ikram M, Jo MH, Yoo L, Chung KC, Nah SY, Hwang H, Rhim $\mathrm{H}$ and Kim MO: Gintonin mitigates MPTP-induced loss of nigrostriatal dopaminergic neurons and accumulation of $\alpha$-synuclein via the Nrf2/HO-1 pathway. Mol Neurobiol 56: $39-55,2019$

39. Kim HJ, Kim DJ, Shin EJ, Lee BH, Choi SH, Hwang SH, Rhim H, Cho IH, Kim HC and Nah SY: Effects of gintonin-enriched fraction on hippocampal cell proliferation in wild-type mice and an APPswe/PSEN-1 double Tg mouse model of Alzheimer's disease. Neurochem Int 101: 56-65, 2016

40. Choi JH, Jang M, Oh S, Nah SY and Cho IH: Multi-target protective effects of gintonin in 1-Methyl-4-phenyl-1,2,3,6-tetrahydropyridine-mediated model of Parkinson's disease via lysophosphatidic acid receptors. Front Pharmacol 9: 515, 2018.

41. Jang M, Choi JH, Chang Y, Lee SJ, Nah SY and IH: Gintonin, a ginseng-derived ingredient, as a novel therapeutic strategy for Huntington's disease: Activation of the Nrf2 pathway through lysophosphatidic acid receptors. Brain Behav Immun 80: 146-162, 2019.

42. Kim DG, Jang M, Choi SH, Kim HJ, Jhun H, Kim HC, Rhim H, Cho IH and Nah SY: Gintonin, a ginseng-derived exogenous lysophosphatidic acid receptor ligand, enhances blood-brain barrier permeability and brain delivery. Int J Biol Macromol 114: 1325-1337, 2018.

This work is licensed under a Creative Commons Attribution-NonCommercial-NoDerivatives 4.0 International (CC BY-NC-ND 4.0) License. 